\title{
An AP4B1 frameshift mutation in siblings with intellectual disability and spastic tetraplegia further delineates the AP-4 deficiency syndrome
}

\author{
Hengameh Abdollahpour ${ }^{1,7}$, Malik Alawi ${ }^{2,3,4}$, Fanny Kortüm ${ }^{1}$, Michael Beckstette ${ }^{3}$, Eva Seemanova ${ }^{5}$, \\ Vladimír Komárek ${ }^{6}$, Georg Rosenberger ${ }^{1}$ and Kerstin Kutsche ${ }^{\star, 1}$
}

The recently proposed adaptor protein 4 (AP-4) deficiency syndrome comprises a group of congenital neurological disorders characterized by severe intellectual disability (ID), delayed or absent speech, hereditary spastic paraplegia, and growth retardation. AP-4 is a heterotetrameric protein complex with important functions in vesicle trafficking. Mutations in genes affecting different subunits of AP-4, including AP4B1, AP4E1, AP4S1, and AP4M1, have been reported in patients with the AP-4 deficiency phenotype. We describe two siblings from a non-consanguineous couple who presented with severe ID, absent speech, microcephaly, growth retardation, and progressive spastic tetraplegia. Whole-exome sequencing in the two patients identified the novel homozygous 2-bp deletion c.1160_1161delCA (p.(Thr387Argfs*30)) in AP4B1. Sanger sequencing confirmed the mutation in the siblings and revealed it in the heterozygous state in both parents. The AP4B1-associated phenotype has previously been assigned to spastic paraplegia-47. Identification of a novel $A P 4 B 1$ alteration in two patients with clinical manifestations highly similar to other individuals with mutations affecting one of the four AP-4 subunits further supports the observation that loss of AP-4 assembly or functionality underlies the common clinical features in these patients and underscores the existence of the clinically recognizable AP-4 deficiency syndrome.

European Journal of Human Genetics (2015) 23, 256-259; doi:10.1038/ejhg.2014.73; published online 30 April 2014

\section{INTRODUCTION}

A group of congenital neurological diseases characterized by severe intellectual disability (ID), absence of speech, and early-onset progressive spasticity leading to spastic para- or tetraplegia has recently been associated with deficiency of different adaptor protein 4 (AP-4) complex subunits. ${ }^{1}$ AP-4 belongs to a family of adaptor proteins consisting of the five heterotetramers AP- 1 to AP-5, which have key functions in vesicle trafficking. ${ }^{2,3}$ APs are composed of two large subunits ( $\beta 1-5$, and either $\alpha, \gamma, \delta$, $\varepsilon$, or $\zeta$ ), one medium subunit $(\mu 1-5)$, and one small subunit $(\sigma 1-5)$, and these mediate different cellular transport steps. The ubiquitously expressed AP-4 complex is composed of the four subunits AP4B1 ( $\beta 1), A P 4 E 1(\varepsilon 1), A P 4 M 1(\mu 1)$, and AP4S1 $(\sigma 1)$ and has a role in trafficking of proteins from the trans Golgi network to endosomes. ${ }^{4,5}$ Mutations in genes encoding different subunits of the AP-4 complex have been associated with autosomal-recessive ID with spastic paraplegia: A homozygous splice site mutation in AP4M1 was found in family members affected by early infantile hypotonia, delayed psychomotor development, inability to walk independently, absent speech, progressive spasticity, and severe ID (MIM 612936). ${ }^{6}$ In two siblings with spastic paraplegia cerebral palsy with profound ID, microcephaly, epilepsy, and white matter loss, a homozygous microdeletion covering part of the AP4E1 gene has been identified (MIM 613744). ${ }^{7}$ Abou Jamra et al ${ }^{1}$ and Najmabadi et $a l^{8}$ described homozygous mutations of AP4B1 (MIM 614066), AP4S1 (MIM 614067), AP4E1, and AP4M1 in individuals with severe ID, growth retardation, stereotypic laughter, progressive spasticity, and inability to walk as the core phenotype. Two Arabic siblings with hereditary spastic paraplegia (HSP), ID, and seizures ${ }^{9}$ were found to carry the homozygous c.664delC mutation in AP4B1. ${ }^{10}$ Thinning of the corpus callosum has been proposed to be a key feature of the AP-4-associated HSP. ${ }^{6,7,10}$ Identification of a homozygous AP4E1 nonsense mutation in twins with HSP and mycobacterial disease suggests a role of the AP-4 complex not only in the neurological but also in the immunological system. ${ }^{11}$ Taken together, the common clinical features in patients with mutations in different subunits of the AP-4 complex indicate that loss of one subunit results in severely impaired complex formation leading to the AP-4 deficiency syndrome. ${ }^{1,2}$ The function of the AP-4 adaptor complex in endosomal trafficking highlights a novel biological mechanism underlying an HSP type associated with severe ID. ${ }^{12}$

Here we report a homozygous 2-bp deletion in AP4B1 in two siblings with severe ID, microcephaly, growth retardation, inability to walk, and absent speech, confirming the common phenotype associated with AP-4 deficiency.

${ }^{1}$ Institute of Human Genetics, University Medical Center Hamburg-Eppendorf, Hamburg, Germany; ${ }^{2}$ University Medical Center Hamburg-Eppendorf, Bioinformatics Service Facility, Hamburg, Germany; ${ }^{3}$ Center for Bioinformatics, University of Hamburg, Hamburg, Germany; ${ }^{4}$ Heinrich-Pette-Institute, Leibniz-Institute for Experimental Virology, Virus Genomics, Hamburg, Germany; ${ }^{5}$ Department of Clinical Genetics, Institute of Biology and Medical Genetics, University Hospital Motol, Second Medical School, Charles University Prague, Prague, Czech Republic; ${ }^{6}$ Department of Pediatric Neurology, Charles University, Second Faculty of Medicine, Motol Hospital, Prague, Czech Republic

${ }^{7}$ Current address: MVZ genteQ GmbH, Hamburg, Germany.

*Correspondence: Professor K Kutsche, Institute of Human Genetics, University Medical Center Hamburg-Eppendorf, Martinistraße 52 , 20246 Hamburg, Germany. Tel: +49 407410 54597; Fax: +49 407410 55138; Email: kkutsche@uke.de

Received 10 December 2013; revised 4 March 2014; accepted 7 March 2014; published online 30 April 2014 


\section{SUBJECTS AND METHODS}

\section{Case reports}

The study was approved by the Ethics Committee of the Medical Chamber of Hamburg (No. PV3802). Written consent was obtained from the parents of patients 1 and 2, including consent to use the photographs in this report.

Patient 1 is a 14-year-old girl (Figure 1a and Table 1) born to nonconsanguineous healthy parents. She was born by natural delivery after medical induction at 41 weeks of gestation; birth weight was $4000 \mathrm{~g}$, length was $52 \mathrm{~cm}$, and occipitofrontal circumference $(\mathrm{OFC})$ was $34 \mathrm{~cm}$. Apgar scores were 10-10-10; there were no perinatal complications. At the age of 3 months, psychomotor developmental delay was noticed. The girl was microcephalic ( -2 s.d.) at the age of 1 year. At 18 months of age, she began to speak a few words. The propositus started to walk at the age of 20 months. At the age of 3.5 years, the patient was admitted to hospital because of duplicated ureter, urosepsis, pelvic duplex, and febrile seizure. Three years later she experienced another episode of febrile seizure, which was controlled by antiepileptic treatment. The patient was referred to one of us (ES) when she was almost 4 years old. She could walk only short distances and spoke short sentences. Microcephaly ( -2 s.d.) and increased tonus of lower limbs were observed. Recent clinical examination at the age of 14 years revealed microcephaly ( -2 s.d.), short stature ( -2 s.d.), club foot, skin hyperpigmentation on the lumbar region, and low anterior and posterior hairline. The patient had spastic tetraplegia with contractures, particularly of the lower limbs, hyperreflexia, and positive Babinski sign; she had been wheelchair-bound since the age of 12 years. Pelvis X-ray revealed valgosity of the hips with bilateral subluxation and acetabular dysplasia. Otologic and ophthalmologic evaluations were normal, similar to conventional and molecular karyotyping as well as biochemical serum and urine tests. She had facial dysmorphism with tongue protrusion, open mouth, widely spaced teeth, gingival hyperplasia, prominent supraorbital ridges, and a broad nasal root. The patient had a shy and anxious character and had been communicating nonverbally since the age of 13 years; she never had stereotypic laughter. She was able to control urinary and anal sphincters and eat without assistance. Electroencephalography (EEG) evaluations revealed a generalized abnormality with a low-frequency brain activity, especially in the centroparietal region. Brain magnetic resonance imaging (MRI) did not show any abnormality.

Patient 2 (Figure $1 \mathrm{~b}$ and Table 1) is the 12-year-old brother of patient 1. Birth was by cesarean section at 41 weeks of gestation because of suspected fetal stress. However, postpartal adaptation was normal with Apgar scores 9-10-10. Birth weight was $3500 \mathrm{~g}$, length was $50 \mathrm{~cm}$, and OFC was $34 \mathrm{~cm}$. Apathetic behaviour was noticed at the age of 3 months. His psychomotor development was delayed: he could walk at the age of 18 months, spoke his first words at the age of 24 months, and had a very short attention span. Physical examination at the age of 2 years showed microcephaly and psychomotor developmental delay. Mild spasticity of lower extremities was noticed with hyperreflexia and Babinski sign. He was able to walk until the age of 7.5 years, after which he could walk only with assistance and had been wheelchair-dependent since the age of 12 . At the age of 10 years, the Achilles tendon on the right side was operated upon because of club foot. Shortly after, febrile convulsions occurred, which led to antiepileptic therapy. X-ray investigation of the hips revealed coxa valga with acetabular dysplasia. Facial dysmorphism was noted with large auricles, synophrys, open mouth, tongue protrusion, prominent nasal root, and mild convergent strabismus. He had a more severe ID than his sister, with very short attention span and inability to communicate. Additional clinical findings were spastic tetraplegia with contractures of the knees, thoracic dextroscoliosis, and lumbar levoscoliosis. He had hyperpigmentation on the right shoulder. He had a shy and anxious character and no history of stereotypic laughter. Latest examination at the age of 12 years revealed mild microcephaly ( -2 s.d.) and short stature ( -2 s.d.). EEG findings were identical to those of his sister. MRI of the brain revealed mild thinning of the corpus callosum in the dorsal region of the splenium.

\section{Whole exome sequencing (WES) and data analysis}

For detailed description of the WES and Sanger sequencing procedures, see Supplementary Methods.

\section{RESULTS}

We performed WES on genomic DNA of patients 1 and 2 and on their mother and filtered for compound heterozygous and homozygous sequence variants in the siblings. No compound heterozygous mutation was identified; however, we detected a 2-bp deletion, c.1160_1161delCA, in the AP4B1 gene (RefSeq NM_006594.2) in two patients in the homozygous state (Figure 2). Sanger sequencing confirmed the homozygous c.1160_1161delCA mutation in exon 7 of AP4B1 in the siblings, whereas both parents were heterozygous (Figure 2). This variant was not found in the databases dbSNP, 1000Genomes, and Exome Variant Server. The AP4B1 variant c.1160_1161delCA identified in this study was submitted to the ClinVar database with the accession number SCV000119895 (http://www.ncbi.nlm.nih.gov/ clinvar/?term $=$ SCV000119895). AP4B1 mutations have already been linked to an autosomal recessive form of ID with spastic paraplegia (spastic paraplegia-47, MIM 614066), which fits well to the phenotype of the siblings. Thus, we concluded that the homozygous frameshift mutation in AP4B1 underlies the neurological disorder in both patients.

\section{DISCUSSION}

We performed WES in two siblings with severe ID, microcephaly, growth retardation, inability to walk, and absent speech, and detected the homozygous 2-bp deletion c.1160_1161delCA in the AP4B1 gene. This mutation results in a frameshift and in the introduction of a premature termination codon ( $\mathrm{p} .\left(\right.$ Thr387Argfs $\left.{ }^{\star} 30\right)$ ). Both parents were heterozygous for the AP4B1 mutation. To date, two other mutations in the $A P 4 B 1$ gene have been identified in individuals with
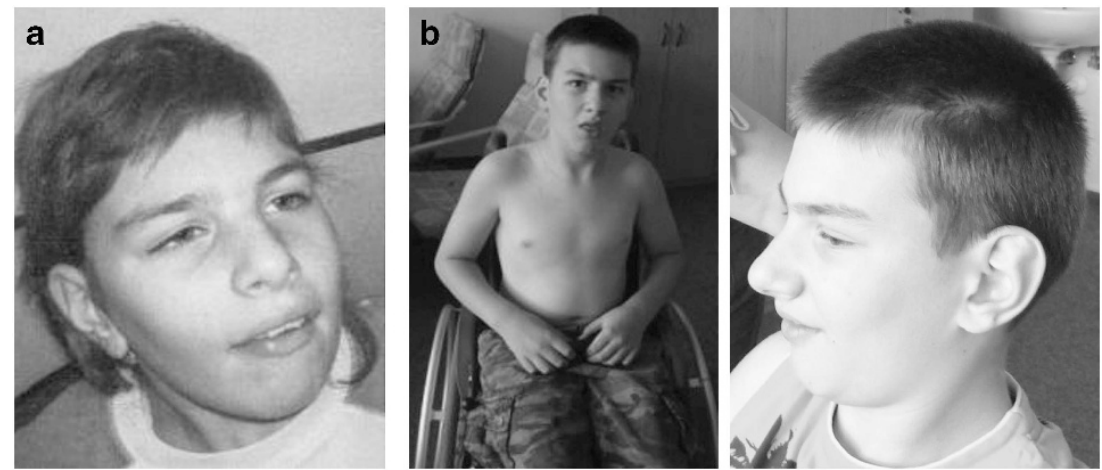

Figure 1 Photographs of the sister and brother with a homozygous AP4B1 mutation. (a) The 14-year-old girl shows an open mouth, prominent supraorbital ridges, and a broad nasal root. (b) Her 12-year-old brother is wheelchair-bound. He has facial dysmorphism with large auricles, synophris, open mouth, and a prominent nasal root. 
Table 1 Clinical features of patients with AP4B1 mutations

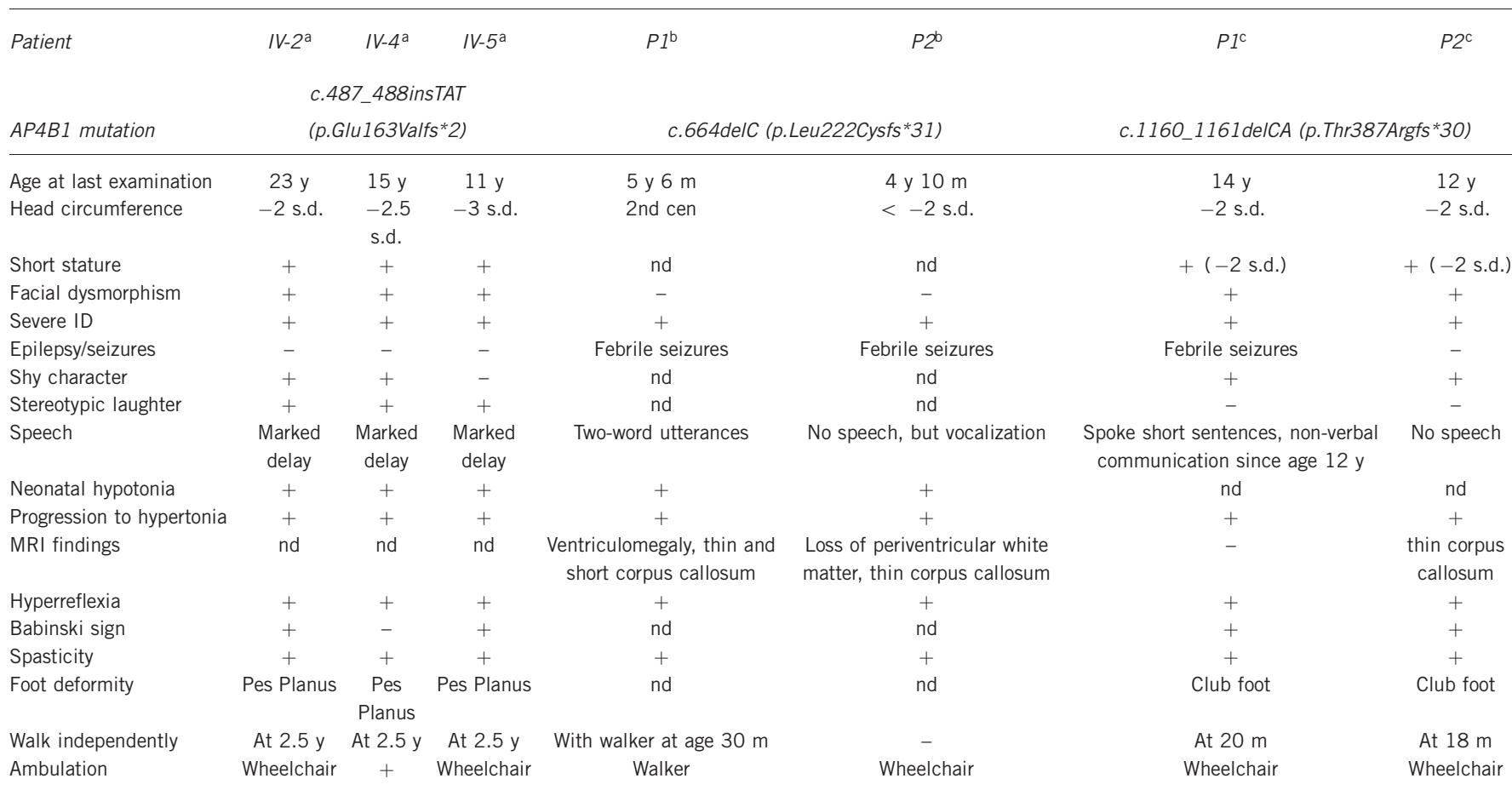

Abbreviations: +, present; -, absent; cen, centile; ID, intellectual disability; m, months; nd, no data; s.d., standard deviation; y, years.

apublished in Abou Jamra et al. ${ }^{1}$

${ }^{\mathrm{b}}$ Published in Blumkin et $\mathrm{a}^{\rho}$ and Bauer et al. ${ }^{10}$

${ }^{\mathrm{c}}$ This report.

a congenital neurological disorder (Table 1): the homozygous 3-bp insertion c.487_488insTAT (p.(Glu163Valfs $\left.{ }^{\star} 2\right)$ is the correct description of the mutation at the protein level $)^{1}$ and the 1 -bp deletion c.664delC (p.(Leu222Cysfs ${ }^{\star} 31$ ) is the correct description). ${ }^{10}$ Remarkably, the three known sequence-level alterations in $A P 4 B 1$ are small rearrangements and most likely represent loss-of-function mutations. This is in agreement with drastically reduced $A p 4 b 1$ transcript levels in fibroblast cells of a patient with the c.487_488insTAT mutation indicating nonsense-mediated mRNA decay. ${ }^{-}$Thus, loss of one AP-4 subunit results in defective AP-4 complex formation leading to impaired endosomal trafficking, especially in the neuronal system.

$A P 4 B 1$ encodes the $\beta 1$ subunit of the AP- 4 complex, which together with AP-1, AP-2, AP-3, and AP-5 constitutes the adaptor protein family. AP complexes $1-5$ are transiently transported onto membranes, where they function as coat proteins to select cargo and shape vesicles. These proteins are ubiquitously expressed in human tissues and have an essential role in vesicle trafficking. ${ }^{2}$ Mutations in genes that code for different subunits of adaptor protein complexes have been described in human disorders. Mutations in AP1S1 and AP1S2 encoding $\sigma$ subunits of the AP-1 complex have been found in patients with MEDNIK syndrome (mental retardation, enteropathy, deafness, neuropathy, ichthyosis, keratodermia) and an X-linked ID syndrome, respectively. ${ }^{13-17}$ The autosomal recessive Hermansky-Pudlak syndrome 2 , which is characterized by platelet defects and oculocutaneous albinism, is caused by mutations in the AP3B1 gene encoding the $\beta 1$ subunit of the AP-3 complex. ${ }^{18}$ Remarkably, heterozygous missense mutations of AP2S1 substituting arginine 15 underlie the familial hypocalciuric hypercalcaemia type $3 .{ }^{19}$ The severe neurological phenotype in patients with mutations affecting components of the AP-4 complex suggests that AP-4 has an important role in human brain development. Indeed, in Ap4b1-deficient mice the somatodendritically localized low-density lipoprotein receptor, AMPA, and $\delta 2$ glutamate receptors were mislocalized in axons, underscoring a function of the AP-4 complex in polarized sorting of these receptors to the somatodendritic domain of neurons. ${ }^{20}$ However, Ap4b1-null mice have an almost normal brain and no neurological defects were seen in this mouse model, ${ }^{20}$ indicating important and non-redundant functions of the AP-4 complex in the human brain.

The phenotype of AP4B1-mutation-positive individuals is quite similar (Table 1). Microcephaly became obvious at the age of 1 year and developed to -2 to -3 s.d. later in life. Where data were available, short stature was present. All individuals had significant psychomotor developmental delay that developed to severe ID; seizures can occur. Five of seven patients could walk independently, and one with assistance. Increased tonus of the lower limbs was noticed between the ages of 2 and 5 years. Spastic para- or tetraplegia with contractures and hyperreflexia was present between the ages of 10 and 14 years. Patients became wheelchair-dependent around the age of 12 years. Marked speech delay or absent speech was noticed in the patients. Brain imaging revealed periventricular white matter changes in two and thinning of the corpus callosum in three individuals; no MRI data were available for three siblings. ${ }^{1}$ The phenotype associated with AP4B1 mutations has been assigned the formal hereditary spastic paraplegia type 47 (SPG47) entry. ${ }^{10,12}$ A thin corpus callosum could likely give a clue to SPG47 and has also been observed in patients harbouring mutations in AP4M1 or AP4E1. ${ }^{6,7}$

In summary, the similar clinical features in patients with mutations affecting different AP-4 subunits underscore the existence of the recognizable AP-4 deficiency syndrome. The phenotype is characterized by microcephaly, severe ID with delayed or absent speech, progressive spasticity leading to wheelchair dependence in early adolescence, and growth retardation. Loss of AP-4 complex 


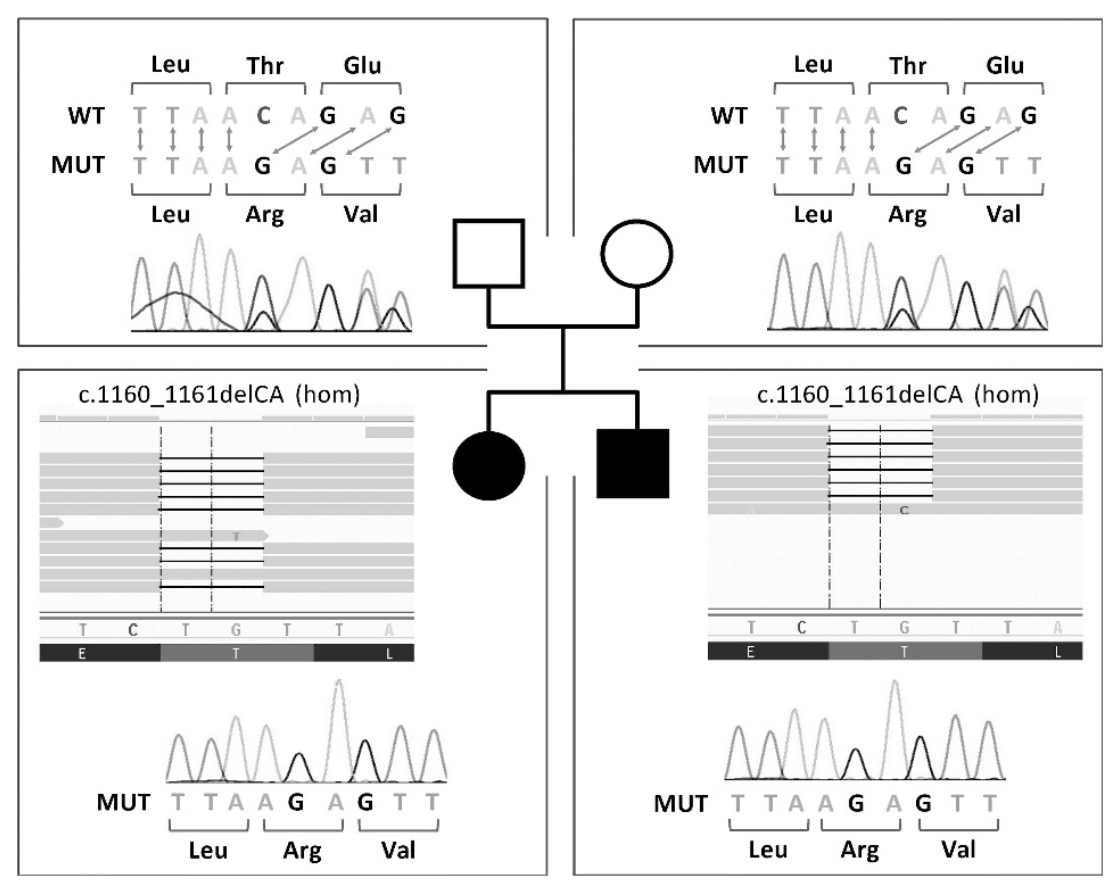

Figure 2 Whole-exome sequencing (WES) and Sanger sequencing revealed the homozygous AP4B1 mutation c.1160_1161delCA (p.(Thr387Argfs*30)) in the two affected siblings. Pedigree of the family with two affected children is shown in the centre. WES identified the 2bp deletion c.1160_1161delCA in the $A P 4 B 1$ gene in both patients (lower boxes). Sequence data are visualized using the Integrated Genomics Viewer) showing the negative strand with complement nucleotides and reverse complement translations. Parts of the Sanger sequencing electropherograms are depicted and demonstrated homozygosity for the frameshift mutation in the siblings; the sequence of the mutant (MUT) allele is given below each electropherogram (lower boxes). The AP4B1 mutation is present in both parents in the heterozygous state. Electropherograms show the Sanger sequencing results, and the sequences of wild-type (WT) and mutant (MUT) alleles are given (upper boxes). Encoded amino acids are indicated in the three-letter code. hom: homozygous.

assembly and functionality in patients with either AP4M1, E1, S1, or $B 1$ mutations is thus the common pathogenetic mechanism underlying the AP-4 deficiency phenotype.

\section{CONFLICT OF INTEREST}

The authors declare no conflict of interest.

\section{ACKNOWLEDGEMENTS}

We are grateful to the family for their interest and participation in this study. We thank Maja Hempel for critical reading of the manuscript. This work was supported by grants of the Deutsche Forschungsgemeinschaft (KU 1240/5-1 to KK, RO 3660/1-1 to GR and KO 4576/1-1 to FK).

1 Abou Jamra R, Philippe 0, Raas-Rothschild A et al: Adaptor protein complex 4 deficiency causes severe autosomal-recessive intellectual disability, progressive spastic paraplegia, shy character, and short stature. Am J Hum Genet 2011; 88: 788-795.

2 Hirst J, Irving C, Borner GH: Adaptor protein complexes AP-4 and AP-5: new players in endosomal trafficking and progressive spastic paraplegia. Traffic 2013; 14: 153-164.

3 McNiven MA, Thompson HM: Vesicle formation at the plasma membrane and transGolgi network: the same but different. Science 2006; 313: 1591-1594.

4 Dell'Angelica EC, Mullins C, Bonifacino JS: AP-4, a novel protein complex related to clathrin adaptors. J Biol Chem 1999; 274: 7278-7285.

5 Hirst J, Bright NA, Rous B, Robinson MS: Characterization of a fourth adaptor-related protein complex. Mol Biol Cell 1999; 10: 2787-2802.

6 Verkerk AJ, Schot R, Dumee B et al: Mutation in the AP4M1 gene provides a model for neuroaxonal injury in cerebral palsy. Am J Hum Genet 2009; 85: 40-52.

7 Moreno-De-Luca A, Helmers SL, Mao H et al: Adaptor protein complex-4 (AP-4) deficiency causes a novel autosomal recessive cerebral palsy syndrome with microcephaly and intellectual disability. J Med Genet 2011; 48: 141-144.
8 Najmabadi $\mathrm{H}$, Hu H, Garshasbi M et al: Deep sequencing reveals 50 novel genes for recessive cognitive disorders. Nature 2011; 478: 57-63.

9 Blumkin L, Lerman-Sagie T, Lev D, Yosovich K, Leshinsky-Silver E: A new locus (SPG47) maps to $1 \mathrm{p} 13.2-1 \mathrm{p} 12$ in an Arabic family with complicated autosomal recessive hereditary spastic paraplegia and thin corpus callosum. J Neurol Sci 2011; 305: 67-70.

10 Bauer P, Leshinsky-Silver E, Blumkin L et al: Mutation in the AP4B1 gene cause hereditary spastic paraplegia type 47 (SPG47). Neurogenetics 2012; 13: 73-76.

11 Kong XF, Bousfiha A, Rouissi A et al: A novel homozygous p.R1105X mutation of the AP4E1 gene in twins with hereditary spastic paraplegia and mycobacterial disease. PLoS One 2013; 8: e58286.

12 Fink JK: Hereditary spastic paraplegia: clinico-pathologic features and emerging molecular mechanisms. Acta Neuropathol 2013; 126: 307-328.

13 Borck G, Molla-Herman A, Boddaert N et al: Clinical, cellular, and neuropathological consequences of AP1S2 mutations: further delineation of a recognizable X-linked mental retardation syndrome. Hum Mutat 2008; 29: 966-974.

14 Cacciagli P, Desvignes JP, Girard N et al: AP1S2 is mutated in X-linked Dandy-Walker malformation with intellectual disability, basal ganglia disease and seizures (Pettigrew syndrome). Eur J Hum Genet 2013; 22: 363-368.

15 Montpetit A, Cote S, Brustein E et al: Disruption of AP1S1, causing a novel neurocutaneous syndrome, perturbs development of the skin and spinal cord. PLoS Genet 2008; 4: e1000296.

16 Saillour Y, Zanni G, Des Portes V et al: Mutations in the AP1S2 gene encoding the sigma 2 subunit of the adaptor protein 1 complex are associated with syndromic $\mathrm{X}$-linked mental retardation with hydrocephalus and calcifications in basal ganglia. J Med Genet 2007; 44: 739-744.

17 Tarpey PS, Stevens C, Teague J et al: Mutations in the gene encoding the Sigma 2 subunit of the adaptor protein 1 complex, AP1S2, cause X-linked mental retardation. Am J Hum Genet 2006; 79: 1119-1124.

18 Dell'Angelica EC, Shotelersuk V, Aguilar RC, Gahl WA, Bonifacino JS: Altered trafficking of lysosomal proteins in Hermansky-Pudlak syndrome due to mutations in the beta 3A subunit of the AP-3 adaptor. Mol Cell 1999; 3: 11-21.

19 Nesbit MA, Hannan FM, Howles SA et al: Mutations in AP2S1 cause familial hypocalciuric hypercalcemia type 3. Nat Genet 2013; 45: 93-97.

20 Matsuda S, Miura E, Matsuda K et al: Accumulation of AMPA receptors in autophagosomes in neuronal axons lacking adaptor protein AP-4. Neuron 2008; 57: 730-745.

Supplementary Information accompanies this paper on European Journal of Human Genetics website (http://www.nature.com/ejhg) 